\title{
Efectos psicosociales de la violencia intrafamiliar en adolescentes
}

\author{
Br. Lineke Ramona Ordoñez Palacios, Br. Erick Yodeman Rivas Peralta, \\ Br. Maryam Tatiana Rizo Escorcia.
}

Coautor:

MSc. Franklin J. Solís Zúniga.

Facultad Regional Multidisciplinaria Estelí.

Recibido 13 de abril 2013-Aprobado 28 de octubre 2013

\section{RESUMEN}

El presente artículo resume una investigación de carácter cualitativa, realizada con adolescentes víctimas de violencia intrafamiliar, atendidas en el Centro Nicaragüense de Derechos Humanos (CENIDH). La investigación se llevó a cabo en el Barrio la Unión ubicado en el distrito tres de la ciudad de Estelí, durante el periodo septiembre a noviembre del año 2012. Con ella se pretendía valorar los efectos psicosociales de la violencia intrafamiliar en adolescentes víctimas. Esta se realizó a partir de un diseño cualitativo desde la perspectiva fenomenológica, con la aplicación de la metodología del estudio de casos. Las técnicas aplicadas para la recolección de la información fueron: la entrevista semi-estructurada y el análisis documental. La información obtenida con las técnicas aplicadas fue analizada en función de los objetivos formulados y según su naturaleza cualitativa. Los resultados más relevantes indican que las vivencias de violencia intrafamiliar han afectado severamente el desarrollo psicosocial de las adolescentes. Entre las afectaciones más notables se encuentran: traumas psicológicos, aislamiento familiar, abandono del hogar, privación al derecho de crecer con sus padres y problemas de autoestima que afectan su autoimagen por la desviación de la conducta normal de un hogar. Las adolescentes sienten que no valen nada, que no son dignas de ser apreciadas y amadas, no se consideran capaces de hacer lo que se les pide en la escuela y sienten que no son buenas en nada de lo que intentan.

Palabras clave: Violencia Intrafamiliar, Adolescencia, Efectos Psicosociales. CENIDH.

\section{INTRODUCCIÓN}

Según Escorcia (2002), en nuestro país, la problemática de violencia intrafamiliar hacia las adolescentes se desarrolla clandestinamente en el seno de un hogar y la comete al menos un miembro de la familia contra otro u otros miembros. Además, señala que dentro de la sociedad muchas veces se justifica y minimiza esta realidad, creando excusas y mitos que tienden a culpar a las verdaderas víctimas, lo que provoca daños irreversibles, tales como: sentimientos de culpa, baja autoestima, incluso la muerte como la peor consecuencia. Toda esta situación dificulta la intervención para erradicarla.

El problema de la violencia intrafamiliar, a nivel nacional se ha incrementado. Según datos de la Comisaria de la Mujer y Niñez de Estelí, diariamente aumentan situaciones de violencia causadas por los padres, madres o tutores que dañan gravemente a las adolescentes, atentando contra su integridad corporal, desarrollo físico, afectivo, intelectual y moral; cuyas expresiones son el descuido, lesiones de orden físico, psíquico y sexual, así como lo son las acciones u omisiones cometidas por los adultos responsables del cuidado cotidiano de niños, niñas y adolescentes en el ámbito familiar.

Las valoraciones anteriores generan preocupaciones en cuanto a los efectos de la violencia intrafamiliar en la personalidad, en las áreas de la vida y creación de expectativas de vida a futuro de las adolescentes y sus familias. Precisamente, la adolescencia es una de las etapas cruciales en la vida humana, en la cual se tiene una conducta orientada a experimentar nuevas formas de sentir y actuar. Esta es una etapa expuesta a toda una serie de problemáticas y cambios de conducta provocados por vivir episodios de violencia que pueden favorecer el desarrollo de afecciones físicas, psicológicas y sociales. 
El interés por el tema surgió al tomar en cuenta que la violencia es un fenómeno social que crece día a día no solo a nivel nacional, sino global, sobre todo en adolescentes. Además, se considera que dará un valioso aporte cualitativo puesto que se han realizado estudios con enfoque cuantitativo, pero no desde el ámbito psicosocial que repercute en las víctimas adolescentes. Por tanto, proporcionará a estudiantes universitarios, interesados particulares, así como al CENIDH, información actual sobre los efectos psicosociales de la violencia intrafamiliar en las adolescentes. Los resultados permitirán orientar y reorientar las políticas y proyectos de desarrollo en pro de la población afectada, asimismo, se creará conciencia social de la gravedad de esta situación.

El estudio se realizó con el objetivo de valorar los efectos psicosociales de la violencia intrafamiliar en las adolescentes del Barrio la Unión de la ciudad de Estelí, atendidas en el CENIDH, en el periodo comprendido de septiembre a noviembre 2012.

\section{MATERIALES Y MÉTODOS}

La investigación se realizó a partir de un diseño cualitativo desde la perspectiva fenomenológica y con la aplicación de la metodología del estudio de casos. Para fines del estudio se seleccionaron aquellos casos de adolescentes víctimas de violencia intrafamiliar registrados en la base de datos del CENIDH.

La muestra estuvo conformada por dos casos de adolescentes identificadas como caso A y caso B, por razones de garantizar la confidencialidad de las participantes. Con el propósito de enriquecer los resultados de la investigación, también se conformó una muestra de expertas en la temática, entre ellas: una Psicóloga, una Trabajadora Social y una Promotora en capacitación y asistencia del CENIDH de la ciudad de Estelí. El proceso de selección de las participantes se realizó mediante el muestreo no probabilístico, de tipo intencional, ya que debían cumplir los siguientes criterios de selección:

- Ser víctima de violencia intrafamiliar.

- Adolecentes con edades de 12 -19 años de edad

- Habitantes del Barrio la Unión de la ciudad de Estelí.

- Que estén registradas en las bases de datos del CENIDH.

- Disponibilidad para formar parte del estudio.

Para la recolección de la información se aplicaron las técnicas de la entrevista semi-estructurada y análisis documental. La entrevista se basó en la elaboración de una guía de preguntas dirigidas con el objetivo de recopilar información de fuentes primarias (adolescentes y expertas en la temática: psicólogas y trabajadoras sociales), sobre las diferentes áreas de vida afectadas en las adolescentes víctimas de violencia intrafamiliar y de esta manera determinar los efectos psicosociales. A través de la entrevista se elaboraron las historias de vida. El análisis documental consistió en la revisión de expedientes de los casos registrados en el CENIDH a través de una guía previamente elaborada. Una vez obtenida la información de los casos de las adolescentes en estudio, en primer lugar se hizo una trascripción fiel de las entrevistas aplicadas, posteriormente se hizo una lectura y relectura de las transcripciones; a partir de las cuales se procedió a la organización y análisis de los datos. El análisis e interpretación de los datos se realizó en base a un sistema de categorías de análisis previamente establecido de acuerdo a los objetivos formulados, y por medio del análisis de contenido.

Con el objetivo de dar mayor confiabilidad al estudio, también se hizo uso del principio de la triangulación para verificar si los datos obtenidos a través de las diferentes fuentes y técnicas de recolección de información guardaban relación o discrepaban entre sí. En este sentido se iba realizando un análisis comparativo de los relatos de las adolescentes y expertas, y posteriormente fueron interpretados. Los resultados se organizaron por ejes temáticos según los objetivos cuyos análisis fueron sustentados con 
los relatos de las informantes y contrastados con la teoría, para luego extraer las conclusiones y plantear las respectivas recomendaciones.

\section{RESULTADOS}

Los resultados reflejan que las adolescentes han vivenciado situaciones de violencia física, psicológica y sexual durante su infancia y adolescencia, producto de un sistema de desigualdad que ha marcado grandes diferencias de poder, donde están más propensas a ser violentadas al considerárseles débiles y desvalorizadas por ser mujer.

Esta situación se manifiesta en el siguiente relato de una de las adolescentes entrevistadas (Caso A): “ $a$ los cinco años mi mamá decidió regalarme a unas personas que ella le trabajaba, yo tengo un mal recuerdo de esto, yo no quería irme a mi papá le dio igual porque él tomaba mucho, maltrataba a mi madre y a mí también y no le importó que a mí me regalaran. Mi mamá justifica esto diciendo que quería lo mejor para mí y que no podía regalar al hijo mayor porque él era varón y a los 2 gemelos".

Opinión de las Expertas: "Las adolescentes mujeres viven aún más violencia por su condición de adolescentes, y también por ser mujeres".

"La violencia intrafamiliar es una forma de ejercer dominio o poder, en muchas ocasiones el que ejerce este tipo de violencia en el seno familiar es el hombre sobre el sexo femenino".

Por otra parte, las adolescentes en estudio han percibido el maltrato como algo natural, una forma de relacionarse con los demás y de resolver problemas. Esto les ha generado serias dificultades tanto a nivel afectivo, como en las relaciones interpersonales. Asimismo, se han visto expuestas a problemáticas psicosociales como el aislamiento familiar y la exclusión social, encontrándose además, que las vivencias de violencia al interior del hogar, han afectado severamente su desarrollo psicosocial.

Expresó una adolescente (Caso B): "Había una señora cercana a mí, quien le pidió a mi mamá que si me regalaba o prestaba para ayudarle, mi mamá por segunda vez lo hizo, al estar con esta mujer tengo un recuerdo muy feo, ella intentó violarme, pues metió sus dedos en mi vagina, no lo llevó a cabo porque yo sali corriendo".

Al respecto las expertas opinaron: "A largo plazo este fenómeno ocasiona daños a la dignidad e integridad física y psíquica de las víctimas de violencia intrafamiliar.

Referente a las acciones implementadas por el CENIDH, mediante la entrevista se constató, que éste forma parte de las instancias de promoción de los derechos humanos, articulando gestiones en la defensa e impulso de los derechos de mujeres, niñez y adolescencia y coordina servicios hacia la población violentada, mediante asesoría legal y psicológica, atención ginecológica y de medicina forense, capacitación y refugio.

\section{DISCUSIÓN}

El estudio de los efectos psicosociales de la violencia intrafamiliar en adolescentes, muestra que la violencia es un problema que involucra aspectos públicos y comunitarios tales como la salud, la educación, la familia, el trabajo y la seguridad. Las adolescentes han vivenciado las problemáticas que encierra la violencia y esta ha afectado su desarrollo y bienestar común, tal y como lo expresan las propias entrevistadas quienes reflejan diversos problemas psicosociales y cómo estos repercuten en su futuro; ya que existen secuelas que serán difíciles de subsanar . 
Estos resultados permiten confirmar lo planteado por Gutiérrez (2008), quien afirma que cuando el joven de hoy, sea un adulto en el mañana y tenga su propia familia, es muy probable que siga ejerciendo la violencia porque ese ha sido el modelo de relacionarse que aprendió; confunde amor con violencia y ve el maltrato como algo natural y de esta manera, se sigue reproduciendo el circulo vicioso de la violencia, que se seguirá transmitiendo de generación en generación.

En este mismo sentido, el estudio comprueba que las adolescentes que viven en familias violentas y que desde niñas han presenciado la violencia de sus padres o padrastros hacia sus madres, tienen mayor probabilidad de aceptar la violencia cuando sean personas adultas, ya que esta les afecta el desarrollo psicológico, dejando secuelas que pueden aparecer en su vida adulta, tal es el caso de una de las adolescentes participante en la investigación, soportando el acoso sexual.

Los resultados de este estudio también son coherentes con los planteamientos de una investigación realizada por Echegaray (2007), en la cual se señala que la afectación psicológica que sufren las adolescentes víctimas de la violencia intrafamiliar, por el maltrato, la ruptura de la pareja (padres), o el abandono, resultó sumamente dañina para la salud mental de las adolescentes involucradas, en ellas se han encontrado problemas de conducta, reacciones de ansiedad, deterioro del autoestima, inhibición social, problemas de rendimiento escolar, temores, fobias y consumo de drogas.

Este autor, también señala que las consecuencias colaterales de esta afectación, se hallan en el orden social y económico. Socialmente el grupo se retrae pierden las perspectivas de sus metas, deteriora su cohesión y los roles se alteran. Por estas circunstancias muchas veces las adolescentes pierden sus estudios, faltan mucho al colegio o simplemente bajan enormemente su rendimiento escolar. La alternativa para estas personas es la intervención sostenida de una terapia psicológica, la intervención de las fuerzas conciliadoras (organizaciones involucradas), el apoyo social orientado a la reinserción escolar que inciten cambios significativos en la conducta de estas adolescentes.

\section{CONCLUSIONES}

El estudio reveló que las adolescentes se han visto expuestas a situaciones de violencia intrafamiliar en etapas cruciales de su vida, siendo éstas la niñez y actual adolescencia. En las historias de vida sobresale la violencia de género, la cual se manifiesta en forma de violencia física, psicológica y sexual. Estas vivencias han originado una serie de problemáticas que han afectado severamente el desarrollo psicosocial de las adolescentes, dejando secuelas que serán difíciles de subsanar.

Finalmente, se comprueba que el hecho de haber sido víctima y testigo de violencia intrafamiliar en la infancia y en su actual adolescencia, puede conllevar a que la adolescente continúe ejerciendo o aceptando la situación de violencia en su vida adulta, puesto que de no trabajarse en el daño causado, se seguirá repitiendo el patrón de conducta que aprendió.

\section{BIBLIOGRAFÍA}

Benavides. M y equipo coordinador (2010), Violencia de género, un mal social. Estelí-Nicaragua: Asociación Miriam.

Cabañas. G. y Subiros. G. (2008), Mujeres contra la violencia. Estelí- Nicaragua: Comisaria de la Mujer y la Niñez.

Echegaray. L. (2007), Efectos Psicológicos de la Violencia Doméstica. Consultado en septiembre 2012 en: http://www.portalpsicologico.org/articulos-psicologia-violencia/efectos-psicologicos-de-laviolencia-domestica-por-luis-echegaray.html

Escorcia. N. (2002), Tratamiento a la problemática de Violencia Intrafamiliar y Sexual. Nicaragua: CENIDH. 